\title{
Analysis of Sigmoidal Equations To Describe the Pulmonary Pressure- Volume Curve in Acute Respiratory Distress Syndrome
}

\author{
Sandra Orfao ${ }^{1}$, Nadine Hochhausen ${ }^{1}$, Ralf Kuhlen ${ }^{2}$ and Dietrich Henzler ${ }^{*}, 1,3$ \\ ${ }^{I}$ Department of Anesthesiology, University Hospital, RWTH Aachen, Aachen, Germany \\ ${ }^{2}$ Surgical Intensive Care, Helios Klinikum Buch, Berlin, Germany \\ ${ }^{3}$ Departments of Anesthesia and Critical Care, Dalhousie University, Halifax, NS, Canada
}

\begin{abstract}
Pulmonary pressure-volume curves (P-V curves) of patients with acute lung injury are commonly analyzed using a parametric algorithm with symmetrical properties. Some of the aspects observed after performing nonlinear regression for two models capable of fitting symmetric, respectively asymmetric data are discussed.

One analyzed aspect was the algebraic complexity of the asymmetric model that does not allow for an estimation of the boundaries of the zone of maximal compliance directly from the parameter estimates in contrast to the symmetric model. Moreover, mathematical evidence is provided.

Using a sigmoid equation for analysis of P-V curves a systematic deviation caused by asymmetrical distribution was encountered, leading to non-robust definitions of lower and upper inflection points. Increasing the number of parameters to fit asymmetric data does not increase physiological expression.

We conclude that some of the drawbacks in using P-V curves may be attributed to imprecise analysis tools. To increase the value of $\mathrm{P}-\mathrm{V}$ curves other forms of mathematical analysis should be investigated.
\end{abstract}

\section{INTRODUCTION}

Quasi-static pulmonary pressure-volume curves (P-V curves) have been used to quantify the elastic properties of the lungs and respiratory system, and therefore to characterize the mechanical behavior of the total respiratory system in the acute respiratory distress syndrome (ARDS). Moreover, several parameters measured directly or indirectly from this curve have been proposed to help adjust the ventilatory settings [1].

$\mathrm{P}-\mathrm{V}$ curves are often recorded during an insufflation of gas that is preceded by expiration to the elastic equilibrium volume. Quantification of the curve consists generally in determining the compliance, i.e. the change of volume per unit of pressure. High compliance is associated with both distension of open alveoli and recruitment of collapsed alveoli [2].

In ARDS the inflation limb of the curve has a sigmoidal shape, and the curve can be described as having three segments: an initial flat segment with very low compliance, followed by a segment with steeper slope, that is, with greater compliance, and a final flat segment at large volumes and high pressures, where a decrease in the compliance can be observed. The transition from one segment to the subsequent one can be more or less abrupt and the two points where these transitions occur are thought to have some special physiological meaning [2]. From the first to the second

*Address correspondence to this author at the Dalhousie University Halifax, Departments of Anesthesia and Critical Care, Queen Elisabeth II Health Sciences Centre, 10 West Victoria, 1278 South Park St., Halifax, Nova Scotia B3H 2Y9, Canada; E-mail: mail@d-henzler.de segment the point is denoted by LIP (Lower Inflection Point) and it is thought to correspond to the pressure at which maximal alveolar recruitment starts [3]; the other point, which can be identified between the second and the third segment, is denoted by UIP (Upper Inflection Point) and is thought to represent the pressure above which maximal elastic distension of the lung parenchyma is approached [3]. The range from LIP to UIP is thought to limit alveolar overdistension and maximize recruitment of alveolar units and is also thought to represent a zone of optimal compliance. In the normal practice, these points are determined visually from the plot of a P-V curve. This leads to a lack of objectivity $[2,3]$.

Several models based on continuous equations have been proposed and they allow parameter estimation according to established analytical techniques [2]. Parameters are therefore determined by fitting the model equation to a P-V curve obtained from investigated subjects. Ideally, the parameters have some physiological interpretation based on mathematical characterization. Otherwise, the evaluation of the pathophysiology of a patient could not be obtained from that model [1].

A sigmoidal form of a P-V model equation,

$$
V(P)=a+\frac{b}{1+e^{-\frac{(P-c)}{d}}}
$$

was proposed by Venegas, Harris and Simon [4]. This equation has 4 fitting parameters $a-d$ with the following physiological meaning: 
$a$ - corresponds to the lower asymptote volume, which approximates residual volume when absolute lung volume and airway opening pressure are used as units. It is expressed in units of volume.

$b$ - corresponds to the vital capacity or the total change in volume between the lower and the upper asymptotes. It is also expressed in units of volume.

$c$ - is the pressure at the inflection point of the sigmoidal curve and also corresponds to the pressure of highest compliance.

$d$ - is proportional to the pressure range within which most of the volume change takes place.

LIP and UIP can directly be determined from the estimation of $c$ and $d$ by the assumption that these points are represented by the point of maximum compliance increase (Pmci) and point of maximum compliance decrease (Pmcd), respectively [4]. The values can be derived from the general expression of the two zeros of the third derivative of equation (1):

\section{Pmci=c-1.317d and $P m c d=c+1.317 d$}

This equation with four parameters reported good agreement of P-V curves obtained from healthy dogs, healthy humans and from patients with ARDS. Furthermore, the authors pointed out that the symmetry of the model used could be the reason for the good agreement. Nevertheless, in the original paper [3], a model to fit asymmetric data was also proposed:

$V(P)=\frac{T L C-B e^{-k P}}{1+e^{-\frac{(P-c)}{d}}}$

Equation (2) included total lung capacity (TLC) and was designed to fit the whole range of lung volumes, whereby former models fitted data only for lung volumes above functional residual capacity [5]. Originally, TLC is the amount of gas in the lungs after a voluntary maximal inspiration. In ventilated patients it often resembles an arbitrary pressure between 25-45 $\mathrm{cmH}_{2} \mathrm{O}$. It was claimed that equation (2) has the important advantage of having a mechanistic basis instead of being purely empirical, even though the more parameters may cause lower parameter sensitivity. However, the conditions of this equation and how a better fit to experimental data can be provided, have not been discussed [6].

The aim of this investigation was to investigate the aspects observed by the implementation of the nonlinear regression for both equations, and to provide some mathematical evidence towards the definition of the conditions under which the asymmetric model may be used.

\section{MATERIALS AND METHODOLOGY}

Pulmonary pressure-volume curves (PV-curves) were obtained from 25 patients with ARDS according to the American-European consensus criteria [7]. The study had been approved by the institutions ethical review board and was conducted according to the Declaration of Helsinki. Informed consent was obtained from a legal surrogate before inclusion in the study. All patients were sedated and paralyzed and ventilated using an EVITA4 ventilator modified by the manufacturer (Draeger Medical, Lübeck, Germany) to perform respiratory maneuvers automatically. The PVcurves were constructed by low flow inflation. For that, the state of complete expiration is held for 5 seconds and afterwards the lung is filled with an extremely low rate of $21 / \mathrm{min}$ until the airway pressure reaches $45 \mathrm{cmH}_{2} \mathrm{O}$. Due to the low flow-rate, the resistance component in the equation of motion is eliminated. Under these quasi-static conditions the recorded pressure-volume relationship was analyzed digitally with a sampling rate of $100 \mathrm{~Hz}$.

In the first part of this article the nonlinear regression implemented for equation (1) is discussed. The second part considers the nonlinear regression for equation (2) followed by a mathematical analysis regarding some analytical properties of this equation. The mathematical relations between these two equations are studied, and the behavior of equation (2) as the pressure $P$ approaches $\pm \infty$ to obtain information about the asymptotes and to establish conditions for stability are investigated. While the lower asymptote represents residual lung volume at total relaxation, the upper asymptote denotes maximum lung volume at TLC, the difference between both representing vital capacity.

Using proc nlin of SAS Inc. V8.2 in a personal computer, both equations were fitted to the $25 \mathrm{P}-\mathrm{V}$ curves available by the Marquardt iterative algorithm. The coefficient of best-fit $R^{2}$ was computed for each case. Furthermore, observedvalues versus predicted-values diagrams were plotted and, to analyze the residuals from the fitted model, a residual-bypredicted plot was computed for each case. The residuals were calculated as actual response minus predicted value.

\section{RESULTS}

\section{Model for Symmetric Data}

Of the $25 \mathrm{P}-\mathrm{V}$ curves available for analysis, convergence problems with the iterative fitting algorithm arose in some cases, but they could be solved by changing the set of initial values. The value of $R^{2}$ was always greater or equal to 99.6 $\%$. In two cases, even though convergence problems were not encountered, the obtained estimates could not be interpreted as having physiological meaning. Since their values fell far out of the interval of measurement (e.g. $a=-872$, $b=7001.7, c=77.8242$, and $d=34.0156$ ), these two cases were omitted from further analysis. The mean values for the parameter estimates are shown in Table $\mathbf{1}$. The graphical components of the analysis are illustrated for one exemplary patient in Fig. (1a-d).

Table 1. Parameters Obtained from the Symmetric Model (Means \pm SD)

\begin{tabular}{|c|c|}
\hline Parameter & Estimates \\
\hline \hline$a$ & $-654.90 \pm 636.27$ \\
\hline$b$ & $3782.36 \pm 1987.36$ \\
\hline$c$ & $26.23 \pm 5.67$ \\
\hline$d$ & $13.73 \pm 4.24$ \\
\hline TLC & $3127.46 \pm 1480.47$ \\
\hline LIP & $8.15 \pm 7.35$ \\
\hline UIP & $44.30 \pm 8.52$ \\
\hline
\end{tabular}

$a, b, c, d$ fitting parameters; TLC total lung capacity, LIP lower inflection point; UIP upper inflection point. 


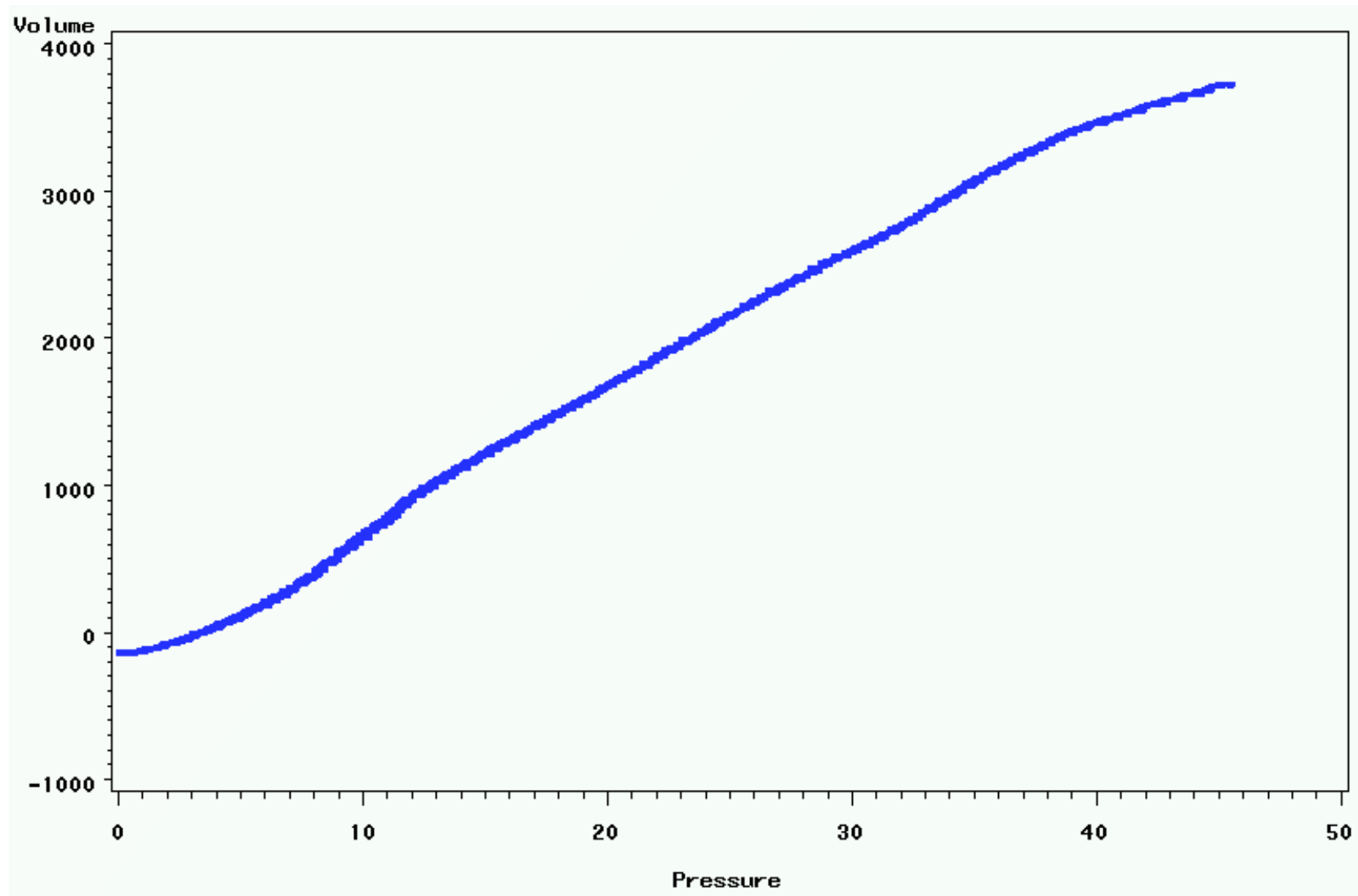

Fig. (1a). Collected source data from low-flow inflation in one exemplary patient with ARDS (Pressure [ $\left.\mathrm{cmH}_{2} 0\right]$ and Volume [ml]). No visual evidence exists that a sigmoidal shaped function would fit the data properly.

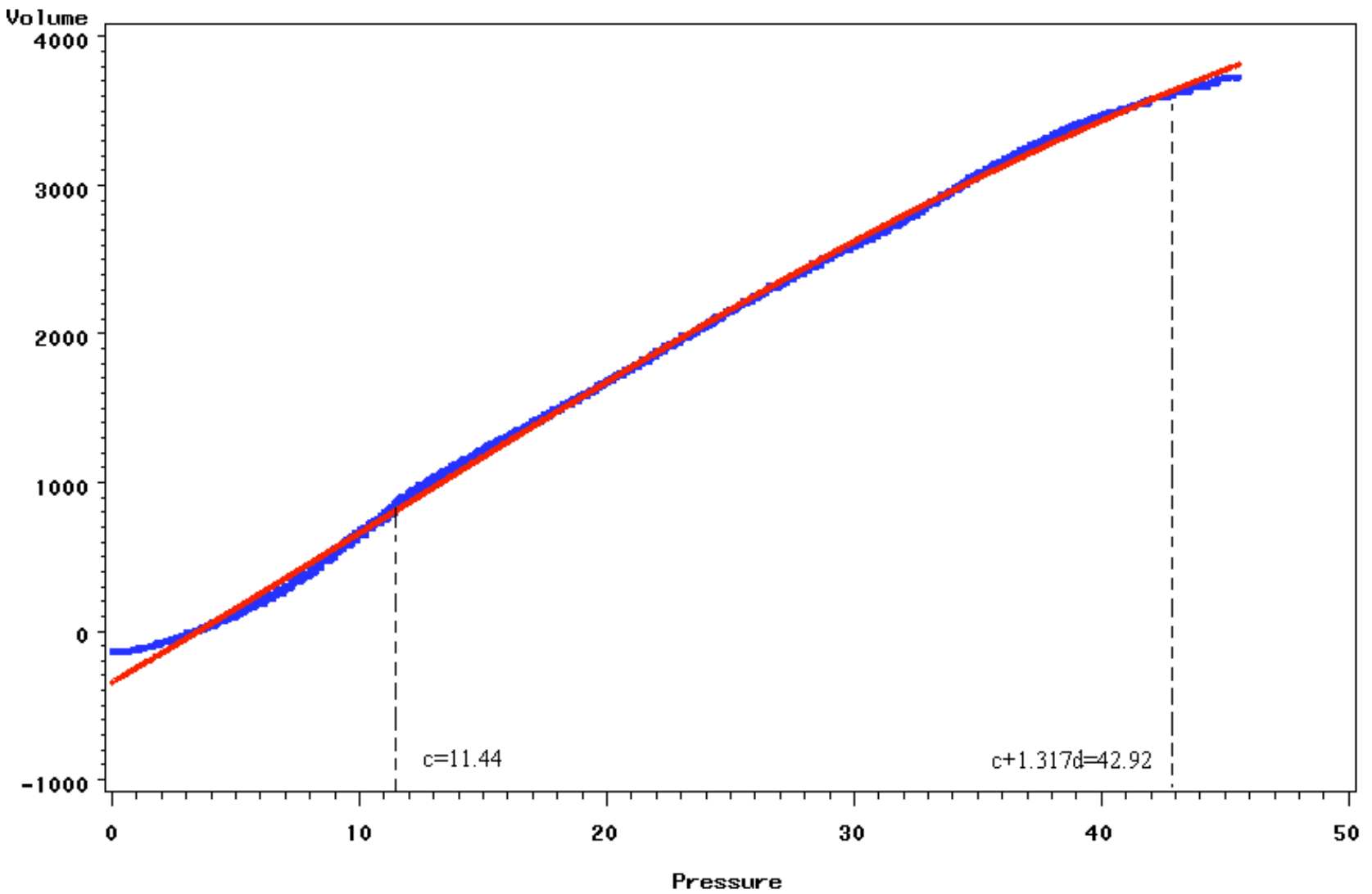

Fig. (1b). Observed-by-predicted plot. The blue curve represents observed PV data, the red curve the fitted equation. A negative value for LIP was obtained (see Fig. 1c). Neither upper nor lower asymptotes can be visually identified in this plot. 


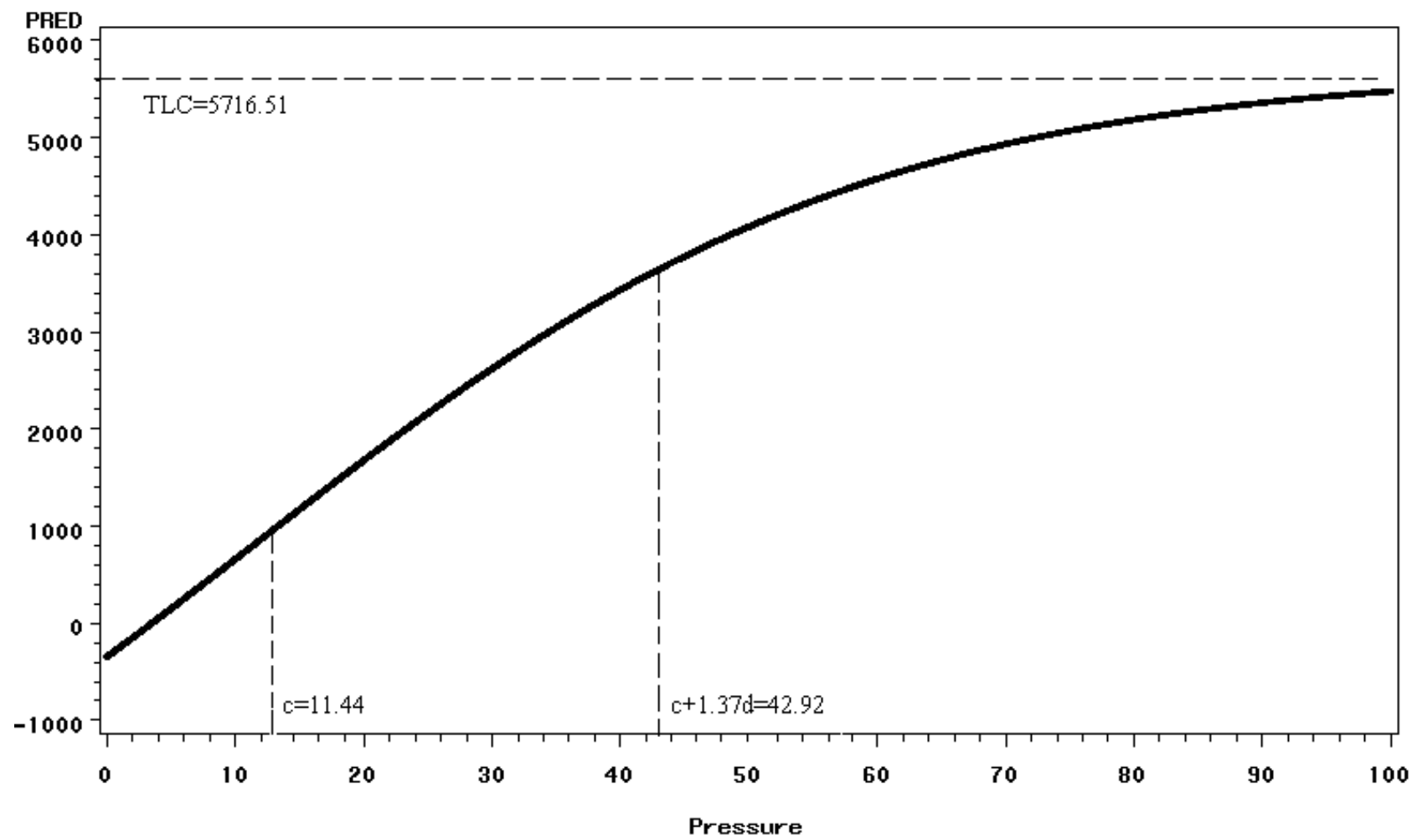

Fig. (1c). Mathematically predicted function with the fitting parameters $a=-4097.5 ; b=9814.01 ; c=11.44 ; d=23.91\left(R^{2}=98.8 \%\right)$. The derived outcomes were Pmci=-20.05; Pmcd=42.931; The value of $T L C=a+b=5716.51$ can be read in the upper asymptote by considering the pressure interval $[0,100]$, but no lower asymptote can be recognized for positive values of pressure.

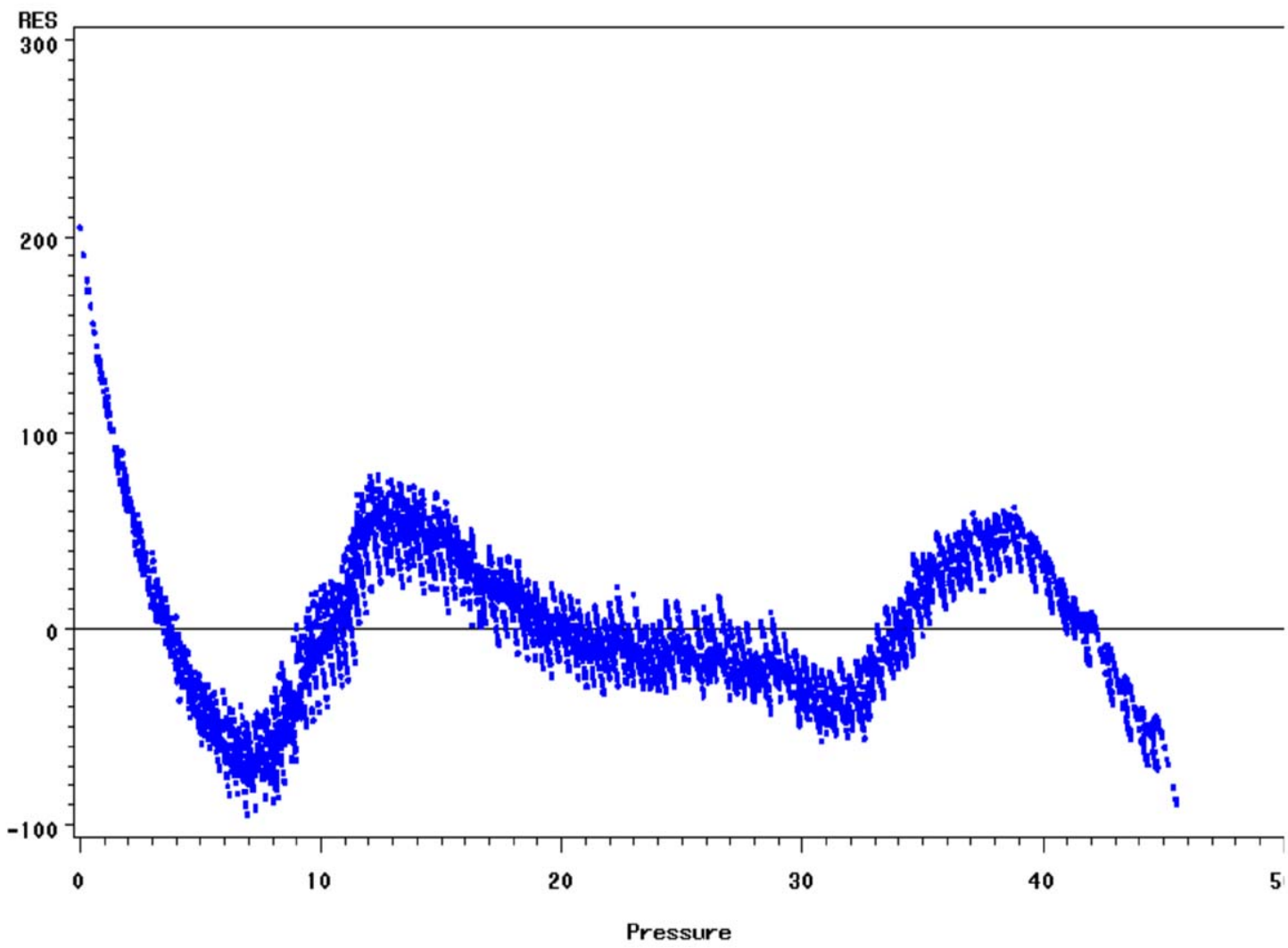

Fig. (1d). Pressure-by-residual plot. Residuals were calculated from the difference of observed and predicted values (from the fitting curve as seen in Fig. (1d)) and are not equidistributed around zero, having a polynomial like shape. (Pressure [cmH2O] and Volume [ml]). 
From viewing the collected data, there is no visual evidence that a sigmoidal shaped function will fit the data properly, but still the algorithm converged and $R^{2}=99.8 \%$. The residuals were not equidistributed around zero, but have a polynom-like shape. This should not be the case if the model had adequate accuracy. No physiological or technical reasons could be found to explain such behavior, which was observed in all cases of this study. The simple implementation of the nonlinear regression to obtain the parameter estimates, therefore, does not seem to be the most adequate method.

\section{Model for Asymmetric Data}

Convergence to the fitting parameters could not be obtained in 8 of the curves, although several sets of initial values were tested. The two cases excluded from the former analysis also belong to this set. Therefore, only the remaining 17 were taken into account for calculating the mean curve. Table 2 summarizes the obtained results.

Table 2. Parameters Obtained from the Asymmetric Model (Means \pm SD)

\begin{tabular}{|c|c|}
\hline Parameter & Estimates \\
\hline \hline$T L C$ & $5158.68 \pm 3076.84$ \\
\hline $\mathrm{B}$ & $6525.28 \pm 3403.36$ \\
\hline $\mathrm{k}$ & $0.092 \pm 0.12$ \\
\hline $\mathrm{c}$ & $19.69 \pm 12.57$ \\
\hline $\mathrm{d}$ & $11.31 \pm 5.41$ \\
\hline
\end{tabular}

$B, k, c, d$ fitting parameters; $T L C$ total lung capacity.
From these estimates, we could not obtain directly the values for LIP and UIP. This means, there is no general expression from the zeros of the third derivative that would allow us doing that, like in the former case. To obtain LIP and UIP using the asymmetric model, one must first find the expression for the first, second and third derivatives of the predicted curve and then calculate approximately the roots of the third derivative. In most cases, we obtained a variable number of roots of complex nature and it is difficult to find general criteria to decide which of these values should be taken. On the other hand, in the original model description, no other procedure to calculate the values for $L I P$ and $U I P$ is given.

For this model, the values of $R^{2}$ remained unchanged and the distribution of residuals had the same structure as with the symmetric model. However, despite convergence, not all the functions had the expected asymptotic behavior. In an exemplary case the obtained parameter estimates were $T L C=2139.5, \quad B=10105.9, \quad k=0.5036, \quad c=30.5035 \quad$ and $d=8.2643$. The obtained function and its derivatives were plotted up to the 3rd order (Fig. 2a-d).

As visualized from the plots the predicted function does not have an asymptote when $P \rightarrow-\infty$; the first derivative, in the interval $(0,45)$ is maximal at $P=0$ and at $P=30.5$; the second derivative has two real zeros and one of them is approximately equal to $31 \mathrm{cmH}_{2} \mathrm{O}$; the third derivative has two real zeros and their values are also very close to 19.3 and 41.3 , respectively.

Comparing the values of these estimates with the ones obtained for this patient from the symmetric model some similarities become apparent. The values for the parameter $c$

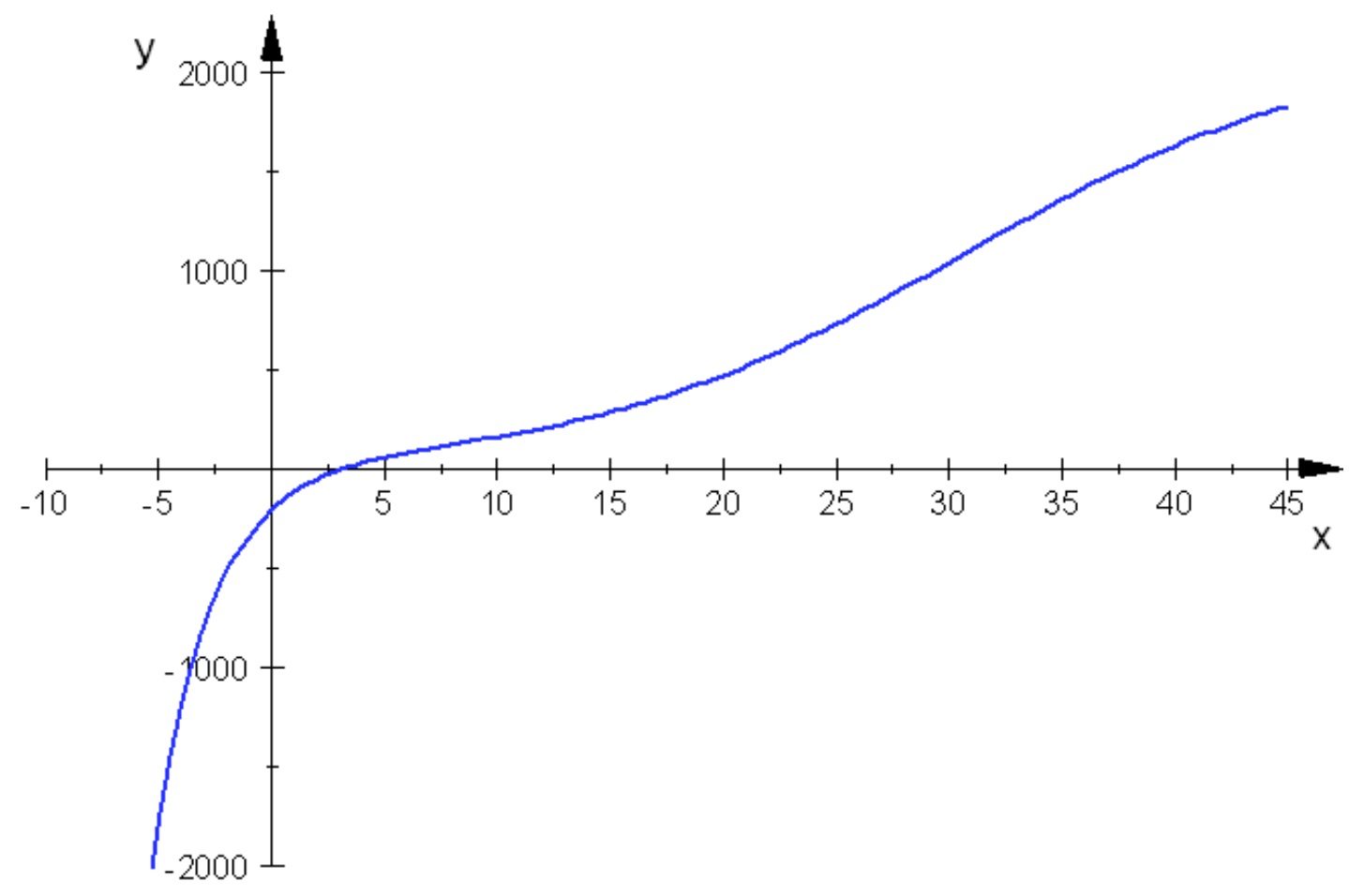

Fig. (2a). After fitting the asymmetric model to the data, the parameters obtained were $T L C=2139.5, B=10105.9, k=0.5036, c=30.5035$ and $d=8.2643$. The predicted function has no asymptotes as $P \rightarrow-\infty$. 


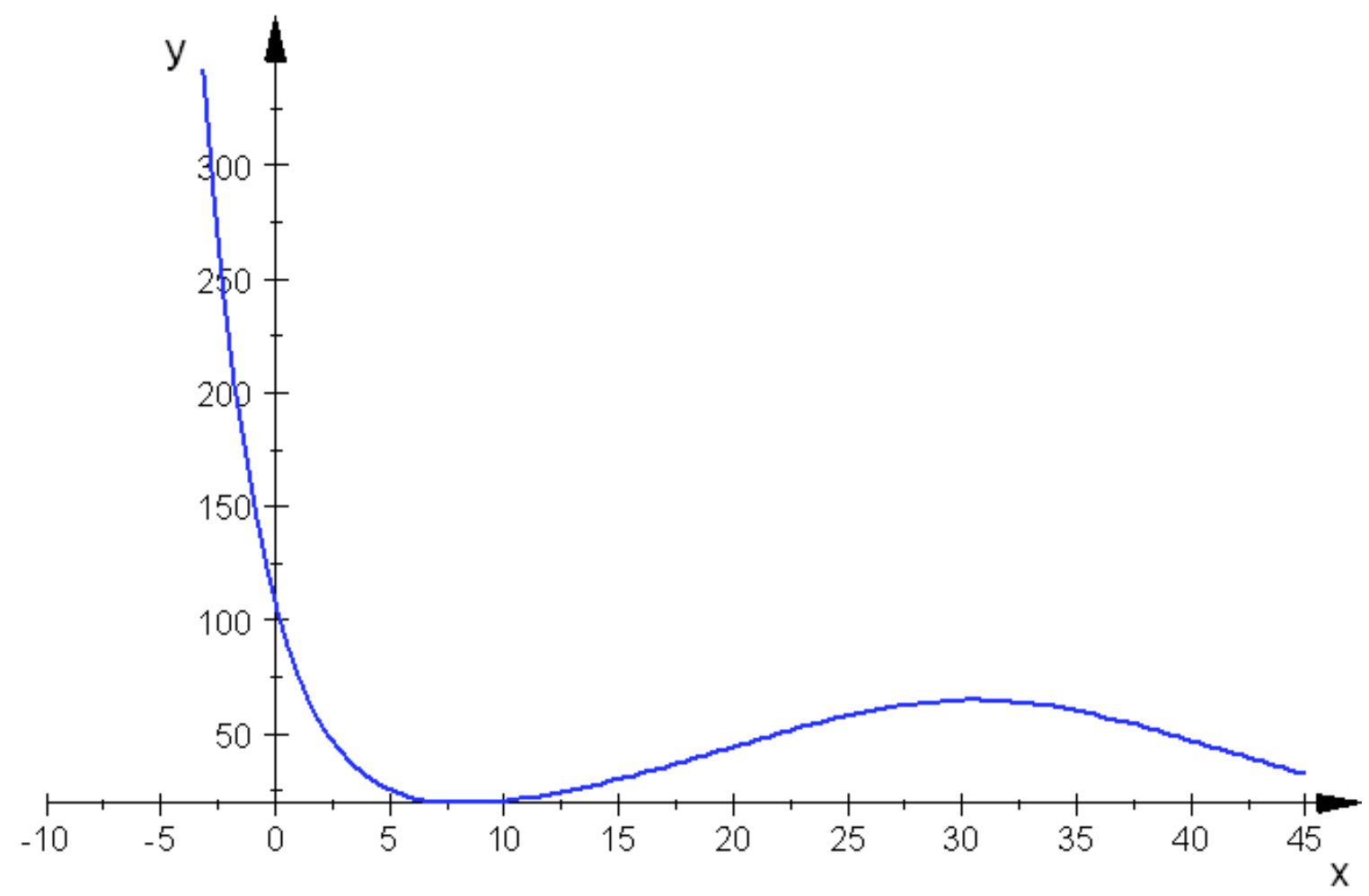

Fig. (2b). The first derivative of the function in $2 \mathrm{~A}$ is maximal at two pressure points $P=0$ and at $P=30.5$ and there is no visual evidence that the derivative is constant in some subinterval of pressure, which would imply that the function is linear in that subinterval.

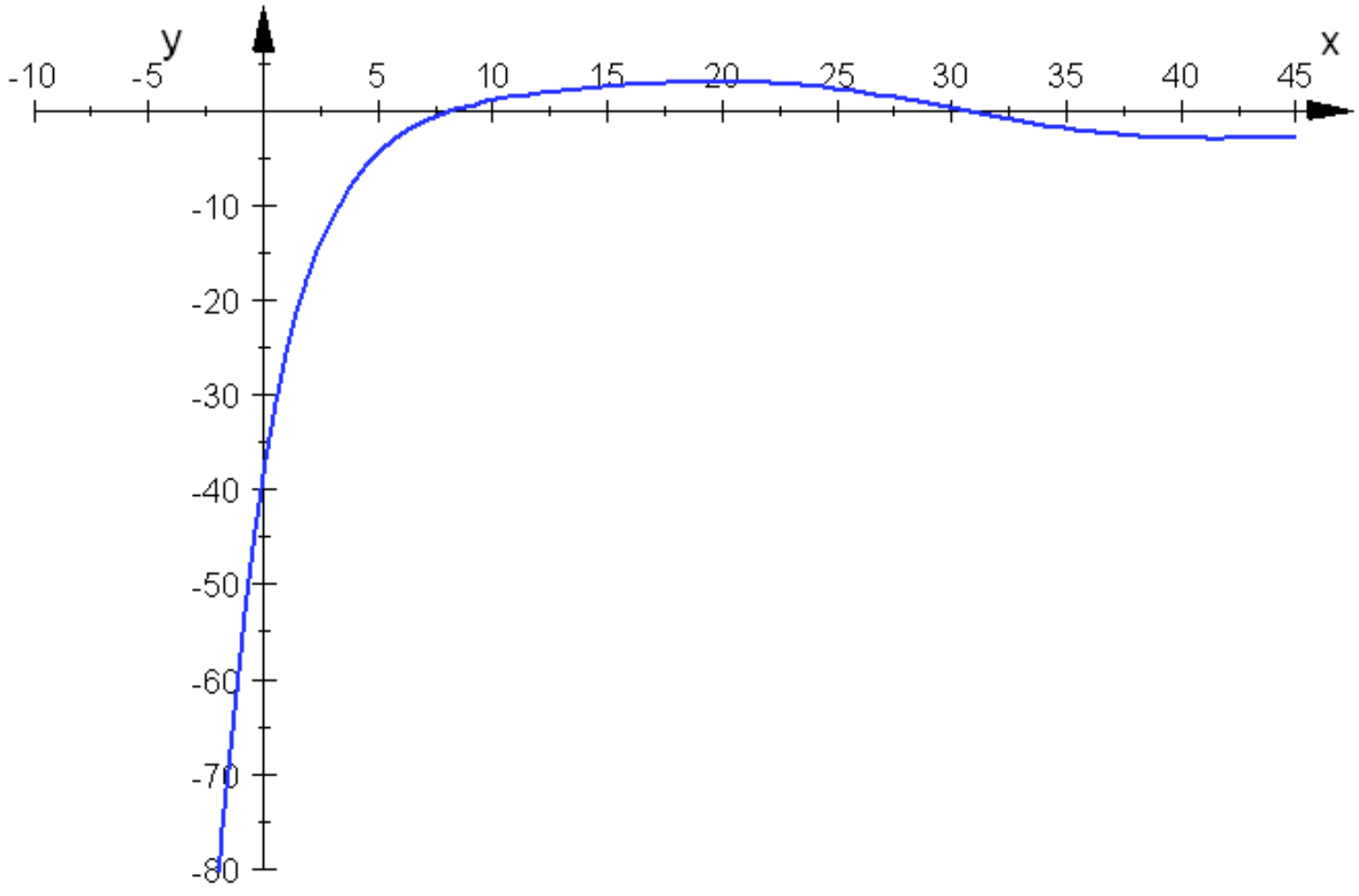

Fig. (2c). The second derivative of the function in $2 \mathrm{~A}$ has two real zeros and changes sign two times, which implies that the original function has two inflection points, one at about 8 and the other about $31 \mathrm{cmH}_{2} \mathrm{O}$ of pressure. 


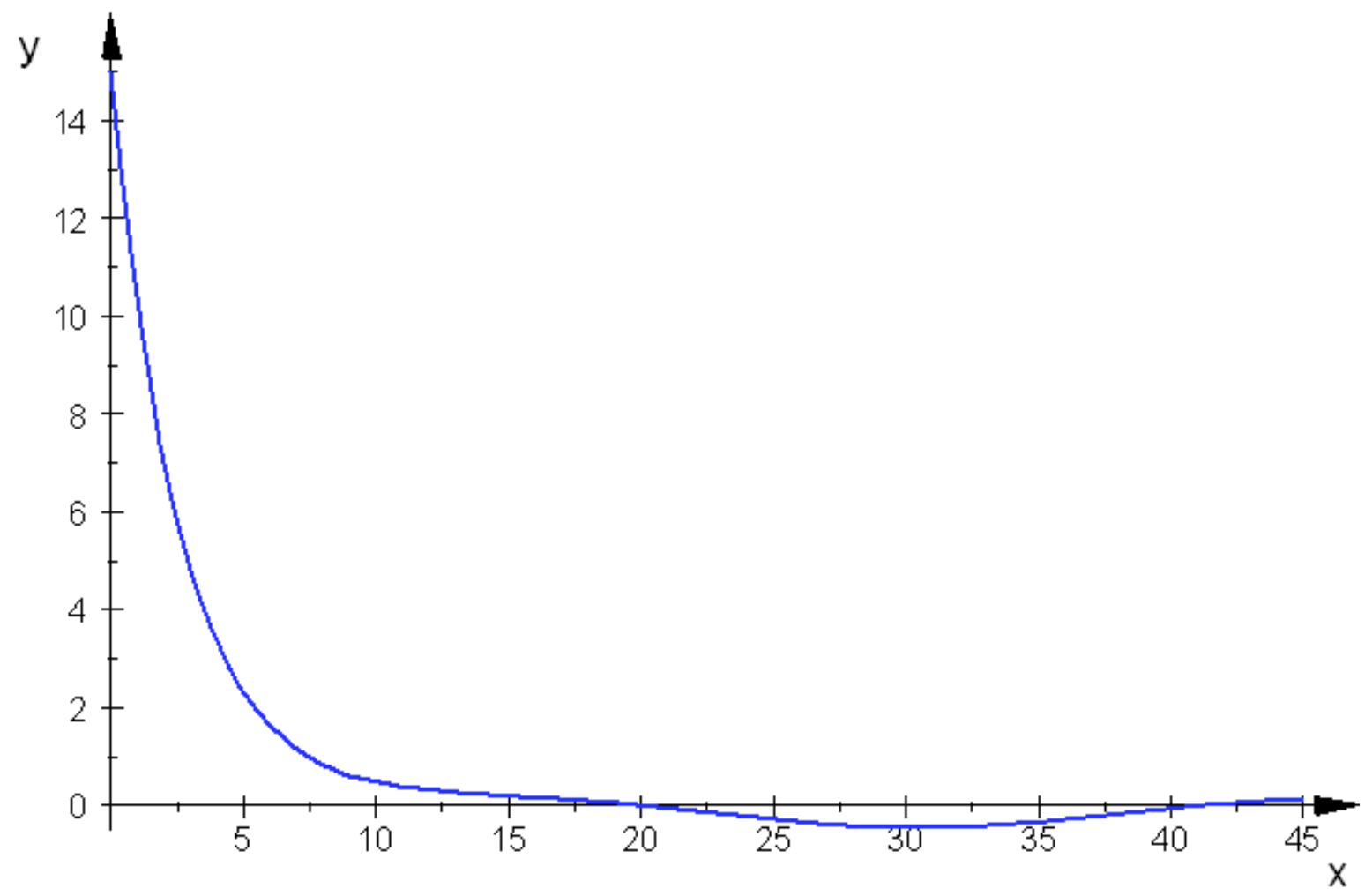

Fig. (2d). The third derivative of the function in $2 \mathrm{~A}$ has also two real zeros, one at 19.3 and the other at $41.3 \mathrm{cmH}_{2} \mathrm{O}$ of pressure.

in both models are very close to each other, and the estimated values for LIP and UIP are also very close to the real roots of the third derivative. However, this was only the case when the estimate of $T L C$ was not close to the estimate of $B$, like in the present example. Therefore, we cannot conclude that the parameter $c$ has the same physiological meaning in both models.

\section{DISCUSSION}

In the analysis of P-V curves of patients with ARDS, we could demonstrate that the analysis of the residual plots should not be omitted. These plots provide information about the accuracy and reliability of the model, meaning the residuals should be equidistributed around zero. In this investigation, the distribution of the residuals appeared to be of polynomial nature, which might give some indication that the model should be improved. Besides, curve fitting appeared impossible in the asymmetric model frequently and 2 times in the symmetric model. Since these models have been developed and validated in animal models of acute lung injury [3], we speculate that the conversion problems represent a mathematical predicament in analyzing diseased human lungs. How can the correction be done without loss of information, in such a way that the parameters maintain some physiological meaning and the typical behavior is preserved?

To have a good estimation of the curve and reliable parameter estimates, we need to measure the curve along the greatest interval of pressure possible. Furthermore, it is important to notice that the pressure range will definitely influence the parameter estimates and the model is not robust against changes of the domain of measurement. In general, and in case of convergence, parameter estimates are ob- tained, but they are probably biased. Nevertheless, some of the estimates do not agree with the expected physiological value. For example, negative values were obtained for LIP and this cannot be the case for patients suffering from ARDS [3].

We could observe that the model for asymmetric data has approximately the same features as the symmetric one under some special conditions. However, the algebraic complexity of the corresponding equation does not allow the finding of the real physiological meaning of the parameters involved by using derivatives, like in the symmetric model. The only parameter that gives directly information about the patient is $T L C$, which corresponds to the upper asymptote in the asymmetric model. The parameter $\mathrm{c}$, which is the true inflection point and also represents the pressure of the greatest compliance, has been similar in the asymmetric model. However, since there is no true inflection point in this model and different values for TLC were obtained, the proposed physiological meaning derived from the symmetric model comes to question. There is no meaningful physiologic explanation why the compliance should increase until the half of the vital capacity is reached and decrease in the same manner beyond.

In some particular cases, both models yielded the same information in regards to the outcomes of interest. However, the asymmetric model was numerically less stable than the symmetric one, leading to physiologically useless data or the loss of certain functions, such as asymptotic behavior.

Some considerations exist from an analytical standpoint. In the classical model of equation (1) by transformation, the following equation can be obtained: 


$$
V=a+\frac{b}{1+e^{\frac{-(P-c)}{d}}}=\frac{a+a e^{\frac{-P}{d}} e^{\frac{c}{d}}+b}{1+e^{\frac{-(P-c)}{d}}}=\frac{(a+b)+a e^{\frac{c}{d}} e^{\frac{-P}{d}}}{1+e^{\frac{-(P-c)}{d}}}
$$

If $T L C=a+b, B=-a e^{\frac{c}{d}}$ and the numerator is replaced by $l / d$ by $k$. the resulting equation is

$$
V=\frac{T L C-B e^{-k P}}{1+e^{-\frac{(P-c)}{d}}}
$$

which is the equation of the asymmetric model.

The asymmetry is caused by the substitution only in the numerator, but both $d$ and $k$ are still related. This fact also has been verified numerically for most of the individual fittings. Both $d$ and $k$ are simply parameters adjusting the scale of the dependent variable. They will not give more information about the phenomena; therefore it is not clear why the number of the parameters should be increased to 5 in this manner.

Assuming $k, d>0$, let us now study the behavior of equation (2) and deduce some particular properties to see what kind of physiological meaning can possibly be obtained from the parameter estimation.

Since $\lim _{P \rightarrow+\infty} V(P)=T L C$

we infer that $V=T L C$ is a horizontal asymptote of the graph of equation (2).

On the other hand,

$$
\lim _{P \rightarrow-\infty} V(P)=\left(-\frac{d B k}{e^{\frac{c}{d}}}\right) \lim _{P \rightarrow-\infty} e^{\left(-k+\frac{1}{d}\right) P}
$$

and we have to distinguish between two situations:

1- If $-k+\frac{1}{d}=0$ then $\lim _{P \rightarrow-\infty} V(P)=a$, hence this is again the Venegas model for symmetric data.

2- If $-k+\frac{1}{d} \neq 0$ then the following two sub cases may occur:

a. If $-k+\frac{1}{d}>0$ then, $\lim _{P \rightarrow-\infty} V(P)=0$ and $V=0$ is the lower horizontal asymptote.

b. If $-k+\frac{1}{d}<0$ then, $\lim _{P \rightarrow-\infty} V(P)=-\infty$ and no lower asymptote exists. (see Fig. 2)

Furthermore, one can ask what happens when $T L C \approx B$, for example $V_{0}$, which has been considered previously [4].

Then equation (2) becomes

$V=\frac{V_{0}\left(1-e^{-k P}\right)}{1+e^{-\frac{(P-c)}{d}}}$

and $\lim _{P \rightarrow \infty} V(P)=V_{0}$.

For the limit as $P \rightarrow-\infty$, we have a similar situation as before: $\lim _{P \rightarrow-\infty} V(P)=-V_{0} k d e^{-\frac{c}{d}} \lim _{P \rightarrow-\infty} e^{\left(-k+\frac{1}{d}\right) P}$

Once again, two cases have to be distinguished:

1-If $\quad-k+\frac{1}{d}=0 \quad$ then $\quad \lim _{P \rightarrow-\infty} V(P)=-V_{0} e^{-\frac{c}{d}}$ and

$V=-V_{0} e^{-\frac{c}{d}}$ is the lower asymptote.

2- If $-k+\frac{1}{d} \neq 0$, the following two sub cases may occur:

a.if $-k+\frac{1}{d}>0$ then $\lim _{P \rightarrow-\infty} V(P)=0$, and $V=0$ is the lower horizontal asymptote.

b. $\quad$ if $-k+\frac{1}{d}<0$ then $\lim _{P \rightarrow-\infty} V(P)= \pm \infty$ is depending on the sign of $V_{0}$. The lower asymptote does not exist.

\section{CONCLUSIONS}

Stability of the asymmetric model is achieved only in the conditions of Venegas' original model. Therefore, in this case parameter estimation provides the same information in both models, e.g., if we calculate the zeros of the third derivative we obtain the same values for LIP and UIP if analyzed in the same pressure domain. From these considerations, we conclude that the asymmetric model has similar properties as the symmetric one. The addition of more parameters to adjust for asymmetry in data does not add to the meaningfulness or change the values of certain parameters one aims to obtain to use this as an important tool. Instead, the resulting analyses are more complex, less stable and the loss of information, such as the lower asymptote, is more likely to occur. Although both models are limited to solutions with some biological significance, some remaining unclearness implies that the underlying physiology is still not completely understood and should be analyzed more critically.

\section{Clinical Implications}

In view of the proposal to set ventilatory parameters according to measurements obtained from the pulmonary pressure-volume curve, there is an urgent need for reliable and objective analysis of these curves. There is still considerable uncertainty about the meaning of pressure-volume relations, which could not be improved in the past ten years [2]. There is evidence that recruitment occurs beyond the lower inflection point [8] and that alveolar collapse is an expiratory phenomenon. The usefulness of the determination of the lower inflection point and the inspiratory pressurevolume curve has been questioned. Worrisome, several commercially available ventilators are equipped with tools to construct the PV-curve. However, the absence of analysis software for these graphically displayed curves or recommendations for changes $n$ ventilator settings might be attributed to the uselessness of existing analysis algorithms.

Moreover, additional information can be obtained from analysis of the whole respiratory cycle [9]. The relations between ventilator settings and intrapulmonary phenomena have been modeled theoretically only [10], which did not lead to consequences in clinical decision making. However, a recent attempt to improve survival of patients with acute lung failure with ventilator settings adapted to very roughly 
calculated estimates of the lower inflection point turned out to be successful [11]. To proceed further in this direction, analysis tools are crucial.

The original model by Venegas et al. is attractive because of its parametric solution, but it is limited due to lack of precision, especially for asymmetric data. Further developments of the model turned out to be more complicated, less applicable and caused the loss of parameters with physiological meaning. It is one thing if there is some underlying theoretical reason behind a particular formulation, to which a priori assignment of meaning can be given to parameters recovered by experiment. But this is not the case in this description of PV curves, which is just simply curve fits. Although not completely unreasonable, it is important to recognize curve fits for what they are: the experimental data "look" like some function which is easily expressed in terms of a small number of parameters.

It is also questionable that the model will be suitable for breath-to-breath analysis of assisted breathing as opposed to static curves in paralyzed subjects. Parametric models are limited in their abilities to provide sufficient information for the clinician to adjust ventilator settings. Moreover, the development of physiologically meaningful parameters is remarkably dependent on the precision of the analysis tools. We therefore speculate that different, non-parametric analysis models could offer more valuable insights into the mechanics of lung inflation-deflation. We are currently investigating a non-parametric approach, which includes not only the shape, but more the change in shape after certain interventions to draw meaningful conclusion.

\section{CONFLICT OF INTEREST}

R. Kuhlen and D. Henzler have received unrestricted research grants from Draeger Medical, Lübeck, Germany, and Hamilton Medical, Rhäzüns, Switzerland, in the past. D. Henzler holds an active unrestricted research grant from Draeger Medical Canada Inc, Richmond ON.

\section{REFERENCES}

[1] Narusawa U. General characteristics of the sigmoidal model equation representing quasi-static pulmonary P-V curves. J Appl Physiol 2001; 91: 201-10.

[2] Jonson B, Svantesson C. Elastic pressure-volume curves: what information do they convey? Thorax 1999; 54: 82-7.

[3] Venegas JG, Harris RS, Simon BA. A comprehensive equation for the pulmonary pressure-volume curve. J Appl Physiol 1998; 84: 389-95.

[4] Harris RS, Hess DR, Venegas JG. An objective analysis of the pressure-volume curve in the acute respiratory distress syndrome. Am J Respir Crit Care Med 2000; 161: 432-9.

[5] Salazar E, Knowles JH. An analysis of pressure-volume characteristics of the lungs. J Appl Physiol 1964; 19: 97-104.

[6] Henzler D, Orfao S, Rossaint R, Kuhlen R. Modification of a sigmoidal equation for the pulmonary pressure-volume curve for asymmetric data. J Appl Physiol 2003; 95: 2183-4.

[7] Bernard GR, Artigas A, Brigham KL, et al. Report of the American-European consensus conference on ARDS: definitions, mechanisms, relevant outcomes and clinical trial coordination. The Consensus Committee. Intensive Care Med 1994; 20: 225-32.

[8] Jonson B, Richard JC, Straus C, Mancebo J, Lemaire F, Brochard L. Pressure-volume curves and compliance in acute lung injury: evidence of recruitment above the lower inflection point. Am J Respir Crit Care Med 1999; 159: 1172-8.

[9] Henzler D, Hochhausen N, Dembinski R, Orfao S, Rossaint R, Kuhlen R. Parameters derived from the pulmonary pressure volume curve, but not the pressure time curve, indicate recruitment in experimental lung injury. Anesth Analg 2007; 105: 1072-8.

[10] Hickling KG. The pressure-volume curve is greatly modified by recruitment. A mathematical model of ARDS lungs. Am J Respir Crit Care Med 1998; 158: 194-202.

[11] Villar J, Kacmarek RM, Perez-Mendez L, guirre-Jaime A. A high positive end-expiratory pressure, low tidal volume ventilatory strategy improves outcome in persistent acute respiratory distress syndrome: a randomized, controlled trial. Crit Care Med 2006; 34: $1311-8$. 